\title{
Could the acarid mite Tyrophagus putrescentiae (Schrank) be used as an environmental thermometer?
}

\author{
C. E. Bowman ${ }^{\mathrm{a}}$
}

${ }^{a}$ Mathematical Institute, University of Oxford, Oxford, OX2 6GG, United Kingdom

\section{ARTICLE HISTORY}

Compiled October 27, 2020

\begin{abstract}
A proof-of-concept for the reconstruction of historical temperatures from museum collections of a commonly-occurring saprophagous mite is recounted. Technological readiness level 3 (TRL3) and TRL4 stage-gates were passed for a multiplex bioassay of ambient temperature using re-purposed laboratory data of setal and body sizes of female Tyrophagus putrescentiae (Acaridae: Astigmata) individuals. Higher temperatures yielded smaller mites with shorter setae. Partial predictive utility of environmental temperature using a composite scale measure was found for mites field-collected in the UK (三TRL5 validation of use). Tyrophagid size in the wild appears individually variable and confounded in the cooler seasons through possible local heating effects (perhaps by virtue of their presence in numbers). Mite shape may change seasonally. Geographically-dispersed detailed data-logging of micro-temperatures in the field together with targeted collection and characterisation of mites at the same locations and measures of their local density is needed in order to advance such biotechnology to any demonstration of effective use all year round (三TRL6) and final use in museum research.
\end{abstract}

\section{KEYWORDS}

Acaridae; Climate inference; Natural history collections-based research; Phenology; Shape; Size

CONTACT C. E. Bowman. Email: bowman@maths.ox.ac.uk 


\section{Introduction}

Mites are ubiquitous. In archaeology and palaeontology their presence offers a wealth of insight as to the past. Museum collections have a huge potential value for research and society (Suzrez and Tsutsui 2004) being an active area of promise (McLean et al. 2016). New, exciting interdisciplinary questions in basic and applied science can be posed (Bakker et al. 2020). In times of disputed climate change, being able to use long-ago preserved zoological repositories to estimate physicochemical-derived measures of the sampled environment from which they arose could be very useful (e.g recent review by Scmitt et al. 2018). Durable remains that stand the test of time are particularly useful e.g. feathers Dosch (2007). However, ecological reconstruction using museum specimens lacking their dates or locations of collection is challenging. In developing any technology to achieve this, nine 'stage-gates' need to be shown, known as Technological Readiness Levels (or TRLs for short; EU Commission 2014). Could that reconstruction technology sought to solve this challenge be the collected animal itself? Could measurements taken from a preserved animal in its own right be considered as an indicative thermometer for the place from which they were collected? That is could a specimen be a biotechnology to infer something about the conditions it lived in?

Prior art for ectotherms is widespread in this field (Atkinson 1994; Kingsolver and Huey 2008). Invertebrates (like other poikilotherms - Barlow 1961; Aguilar-medrano et al. 2011) show temperature-driven size changes with latitude (c.f. Bergmann's rule where cold climes $\rightarrow$ larger size; $\equiv$ TRL1). Given published reports for a temperature-size cline in mesostigmatids (Seeman and Nahrung 2018; $\equiv$ TRL2), commonly found astigmatid mites should be no exception. This paper pursues this proposition using durable chitinous structures. Krzeczkowski (1962) already outlined temperature effects on body size in Tyrophagus noxius $\left(=\right.$ T. putrescentiae) over the range $14^{\circ}-31^{\circ} \mathrm{C}$. Houck and OConnor (1966) found that the highest temperature resulted in the smallest mean body size in hemisarcoptids reared at $15^{\circ}, 24^{\circ}$ and $30^{\circ} \mathrm{C}$. For any widespread use of an animal's scale as a thermometer, one needs: a cosmopolitan species (say the easy-to-collect acarid Tyrophagus putrescentiae, Figure 1); a species with a short life-cycle so that proximate temperature rather than climate can exert 
an effect; and, say by measuring both the changes in body size and the changes in the length of multiple characters such as acarine dorsal setae, the opportunity of developing a potential multiplex bioassay. Using multiple characters for predictions is usually a better tactic than relying upon conclusions from taking just single measures.

The general aim of this paper is to provide a practical exemplar showing the effectiveness (or not) of using measured slide-preserved mite specimens themselves as a basis for an environmental thermometer. If successful other acarologists can then try to extend and deploy the technology for their particular aims. The underlying concept is to first develop a predictor of environmental temperature from body size and setal lengths re-purposed from laboratory-based temperature manipulations of T. putrescentiae (三 TRL3). Then, to validate this (with laboratory specimens $\equiv$ TRL4) before testing it out in estimating the environmental temperature for independently collected field specimens where their location and date is known. This assumes that mites in the wild respond fundamentally in the same non-linear way to temperature as in the laboratory. Of course, this may not be the case. In the wild, this mite prefers living in habitats that have high temperatures (for example, decomposing organic matter, rodent borrows, and bird nests). Furthermore, it is extremely common In human-related habitats, where the temperature, on average, is higher than the temperature in natural habitats. However, its body's response to these different temperatures, i.e. being larger at lower temperatures, is unlikely to be anymore larger or any less larger at that particular temperature just because the mite is in this specific biome or that different biome. If there was an intrinsically different response per biome then it would render many laboratory life history studies of astigmatids investigating temperature effects like Cunnington (1984) of limited value. Next this papers seeks to compare these field mite-derived estimates to UK meteorological data for the temperature of their proximate environment at those locations ( $\equiv$ TRL5). If successfully validated, next to extend this to a test against worldwide field-collected specimens and then to what this might mean geographically regarding variability in astigmatid mite response (三 TRL6). Eventually to pose such a mite as an environmental thermometer for use in diverse international museum collections (三 TRL7) and environmental reconstructions of the past (三 TRL8/9). 


\section{Materials and methods}

The data on Tyrophagus putrescentiae individuals described by Shepherd (1982) was available to the author from Dr Donald A Griffiths. This basis comprised 50 female specimens each of 3 laboratory populations (labeled T2, T13, T58), each reared at 3 temperatures $\left(10^{\circ}\right.$, $\left.15^{\circ}, 25^{\circ} \mathrm{C}\right)$. Details of the original populations are given in Table 1 . Griffiths (1979) indicates these cultures represent isolated breeding groups of T.putrescentaie - recent speculation is that one group might actually be Tyrophagus fanetzhangorum. The cultures at $10^{\circ}$, $15^{\circ}$ and $25^{\circ} \mathrm{C}$ had been originally setup each from several hundred eggs taken from the corresponding $20^{\circ} \mathrm{C}$ 'stock' culture for that population (at the now defunct Pest Infestation Control Laboratory, London Road, Slough, UK), that themselves had been maintained at $90 \% \mathrm{RH}$ on a yeast and wheatgerm mix for many years. All cultures had been run at $90 \%$ RH for more than a year at their respective temperature before sampling by Shepherd (1982).

Included with the dataset were samples of 50 female adult mites reared at $10^{\circ}, 15^{\circ}$, $20^{\circ}$ and $25^{\circ} \mathrm{C}$ and $90 \% \mathrm{RH}$ under reduced gene pool selection (these are labeled as PT2, PT13, PT58). Reduction in gene pools was achieved by randomly selecting one male and one female mite from the stock maintenance culture for each population and allowing them to cross (separately). The resulting eggs were reared, again only one male and one female were randomly allowed to cross and so on to the fourth filial generation for each population separately. At that point, the resultant eggs were allowed to develop and transference to different temperature regimens were carried out. Mites were cultured for up to 35 generations at their respective temperature before sampling by Shepherd (1982). Note that this changed temperature exposure was shorter than that used in the original non-reduced gene pool group.

Idiosomal index (Lynch 1989) and the lengths of dorsal setae (D1, D2, L2 - Griffiths et al. 1990) all in $\mu \mathrm{m}$ had been measured throughout. The original aim of Shepherd (1982) was to measure the stability of diagnostic setal ratios classifying isolated breeding groups. The latter aim is not investigated, nor is the original Shepherd (1982) data put into the public domain. 
As a means to a different end, this dataset was re-purposed and re-formed into:

a 'Training data set' (structured by population) for TRL3 stage-gate, assembled as individuals from

- $\mathrm{T} 2 \_10^{\circ}, \mathrm{T} 2 \_15^{\circ}, \mathrm{T} 2 \_25^{\circ} \mathrm{C}$;

- $\mathrm{T} 13 \_10^{\circ}, \mathrm{T} 13 \_15^{\circ}, \mathrm{T} 13 \_25^{\circ} \mathrm{C}$;

- $\mathrm{T} 58 \_10^{\circ}, \mathrm{T} 58 \_15^{\circ}, \mathrm{T} 58 \_25^{\circ} \mathrm{C}$;

representing 'steady-state' female tyrophagid sizes at a temperature, $N=450$, and,

a 'Validation data set' (structured by population) for TRL4 stage-gate, assembled as individuals from

- PT2_10, PT2_ $15^{\circ}, \mathrm{PT} 2 \_20^{\circ}$; PT2 $25^{\circ} \mathrm{C}$;

- PT13_10, PT13_15 ${ }^{\circ}$ PT13_20 $0^{\circ}$ PT13_25 ${ }^{\circ} \mathrm{C}$;

- PT58_10 $0^{\circ}$ PT58_15 ${ }^{\circ}$, PT58_20; PT58_25 ${ }^{\circ} \mathrm{C}$;

representing (reduced gene pool) female tyrophagid sizes after a relatively more 'recent' exposure to (any change in) temperature, $N=600$. Note that: PT2 $220^{\circ}$; PT13_20 ; PT58_20 ${ }^{\circ} \mathrm{C}$, were thus samples closest to the 3 original 'stock' cultures.

A diverse variety of preserved slide material of independently determined T.putrescentiae slides was accessed by the author from the UK and Overseas (totalling 138 female plus 100 male specimens from 67 different locations and a variety of dates over the summer and winter season over different years). Twenty seven specimens (5 male, 22 female) of Tyrophagus nieswanderi at two locations (one in UK and one overseas) were also available. Drawings of each mite and its setae had been made from such cleared mounted specimens using Nomarski interference phase-contrast microscopy with a drawing tube and micrometer scale. These drawings were scanned using a HP OfficeJet Pro 8720 and digitised measurements (of IL, D1, D2 and L2) made using ImageJ 1.51s ex National Institutes of 'Health USA (http://imagej.nih.gov.uk/ij). Left and right setal lengths were averaged if both were available. These were to be used as the 'Test data sets' (for TRL5 and TRL6 stage-gates). 
The UK-collected individuals (with a full set of length measurements, location and collection date) in the Test data sets were annotated with Latitude and Longitude using GoogleMaps $^{T M}$. The meteorological source for the UK was the Met Office. The temperature data for 'Historic Stations' can be found at https://www.metoffice. gov.uk/research/climate/maps-and-data/historic-station-data

The maximum monthly temperature for the month in which the specified day of the UK mite collection in the test data set, abstracted for the three closest stations (by the shortest road route) to the geographic location of each measured mite in the test dataset was averaged as a summary reference temperature for that specimen. This is denoted as AvMax. In compliance with EPSRC's open access initiative, the 'all-complete' TRL5 test dataset of 91 T.putrescentiae individuals (51 females plus 40 males) is available from https://doi.org/10.5287/bodleian:0005Y00Y0. The monthly window was chosen to approximately match the typical egg to adult generation time of T.putrescentiae (106.0 days at $10^{\circ} \mathrm{C} ; 32.2$ days at $15^{\circ} \mathrm{C} ; 15.8$ days at $20^{\circ} \mathrm{C} ; 9.4$ days $25^{\circ} \mathrm{C} ; 7.2$ days at $30^{\circ} \mathrm{C}$; - Sánchez-Ramos and Castalñera 2001).

All acarine measurements were $\log _{e}$ transformed before use to allow for allometry. Analyses were done in Excel2011 and R version 3.4 .4 (2018-03-15). Heat-maps used Graphis 2.7.3. Ordinations used singular value decomposition (SVD) of covariance matrices $\Psi$ (i.e. $=$ sums of squares and cross-products $(\mathrm{SSCP})$ of the transformed data; it was not standardised into $z$-scores for each variable).

\section{Results}

This will be structured by TRL phase.

As expected, Tyrophagus putrescentiae mites exposed to higher temperatures were smaller with shorter setal lengths on average than mites exposed to lower temperatures (Table 2). Observed variation is due to inter-individual variation between mites and to random mea- 
surement error. Bowman (2020) gives broadly similar mean and standard deviation idiosomal index (IL) figures for independent samples of laboratory cultured: Tyrophagus putrescentiae ['A'] T13 as 188.49, 35.24 $\mu \mathrm{m}$; and, Tyrophagus putrescentiae ['B'] T9 as 210.92, $16.11 \mu \mathrm{m}$, respectively. Figure 2 shows congruence of comparison over the 4 measures (IL, D1, D2, L2) i.e. a consistent trend in overall scale difference (if present) between training (TRL3) and validation data sets (TRL4) within each population over temperature. Only 16 comparisons out the 36 between each population/temperature pair indicate a significant difference at the 2-sided 5\% level (no multiplicity adjustment). The sign of the mean difference at any one temperature between the two data sets is not consistent across the populations. Of these differences only four were a relative drop in size between the training and validation data sets. So, although short-term temperature exposure (and a reduced gene pool) does not induce any consistent general pattern of size changes markedly distinct from basic long-term temperature exposure, there is some evidence that prolonged exposure to a temperature might engender a slightly different scaled mite than any short exposure. Perhaps there is a small degree of genetic adjustment in the process used? Nevertheless, the use of the PT2; PT13; PT58 data set as a laboratory validation of any thermometer formed from the T2; T13; T58 data set is not strongly contradicted for the purposes of this paper's proposition.

\section{TRL3 - experimental proof of concept}

\section{Forming a composite measure from multiplex assay}

Given the results of Klimov et.al.(2004) and in particular those of Houck and OConnor(1966) that different Hemisarcoptes spp. show different temperature responses in different morphological measures, allowance of relative variation between structures at different temperatures should be made. A SVD decomposition of the average within-culture within-temperature covariance matrix over the $\log$ transformed measures in an augmented $(N=450+150=600)$ training data set, i.e.

$$
\frac{1}{12} \cdot\left(\Psi_{P T 2 \_20}+\Psi_{P T 13 \_20}+\Psi_{P T 58 \_20}+\Sigma_{i=[10,15,25]}\left(\Psi_{T 2 \_i}+\Psi_{T 13 \_i}+\Psi_{T 58 \_i}\right)\right)
$$


yielded an eigen analysis showing only one major component (Table 3). This component is essentially equi-weighted on setal lengths but with a difference in importance for idiosomal index IL. The second component captures a minority of the sample variation and contrasts lengths IL, D1 and D2 which are generally aligned antero-posteriorially with setal length L2 which is aligned laterally. The first component of this multiplex assay captures most of the morphological variation within any one population and temperature (after allometry allowed for). Training dataset augmentation (with the $20^{\circ} \mathrm{C}$ values) was to ensure all possible temperature effects on covariation were allowed for without majorly compromising the use of the validation data set.

The measurement data in the training set $(N=450)$ can then be accordingly collapsed into a single species-specific multiplex surrogate measure 'THETA' $\Theta$ as follows for each mite

$$
\begin{aligned}
\Theta=[-0.1804933 * L N(I L)] & +[-0.5726658 * L N(D 1)] \\
+ & {[-0.5586939 * L N(D 2)]+[-0.5721338 * L N(L 2)] }
\end{aligned}
$$

\section{Building the thermometer}

The $\Theta$ surrogate in (1) behaves linearly with temperature $T$ across the $10^{\circ}, 15^{\circ}, 25^{\circ} \mathrm{C}$ range for each population in a similar way (result not shown) and can be summarised overall by a simple regression $R^{2}=0.9161$ giving a prediction equation

$$
\hat{T}=[31.683 * \Theta]+256.15
$$

for any new mite (based upon the training data $N=450$ ). The hat over a symbol signifies 'estimate'. Clearly, extrapolating this would infer values less than zero ${ }^{\circ} \mathrm{C}$ for very big mites and beyond lethal temperatures for very small mites. So, assuming that T.putrescentiae cannot grow at or below $6^{\circ} \mathrm{C}$, nor at or above $36^{\circ} \mathrm{C}$ (abstracted from Sánchez-Ramos and Castalñera 
2001), and a logistic relationship between $\Theta$ and actual temperature $T$ of:

$$
T-6=\frac{30}{1+e^{-(a . \Theta+b)}}
$$

Then this can be fitted to the training data set $(N=450)$ by the linear regression of $\Theta$ as:

$$
a . \Theta+b=\ln \left[\left(\frac{T-6}{36-T}\right)\right]
$$

yielding $\hat{b}=2.43014$ and $\hat{a}=17.57613$ estimates (Figure 3).

The performance of this against the training data set itself $(N=450)$ is shown in Table 4 , note the very close agreement in the mid-range for the $15^{\circ} \mathrm{C}$ data and low standard deviation $\sigma$ values for predictions showing a decent fit. There is a modest tendency for large mites to have a little too high a predicted value, and for small mites to have a little too low a predicted value. Despite this being a 'self-fulfilling prophecy' assessment, the TRL3 stage-gate is past sufficiently.

\section{TRL4 - technology validated in lab}

Calculating $\Theta$ using equation (1) for the mites in the validation data set $(N=600)$ and feeding into the estimated prediction equation (2) yields the results in Figure 4 and Table 5 . Standard deviation $(\sigma)$ values for predictions remain low. The results are consilient with TRL3 and the scale differences detected in Figure 2 suggesting no untoward extra variation has been introduced. There is some evidence of predictions being a little lower than actual for small mites and a little higher than actual for large mites, however the modest standard deviations infer it is worth proceeding to try the equation out in the field. Although by virtue of the curved relationship and the inter-individual variation, the minimum and maximum predicted values can be quite large (at $10^{\circ} \mathrm{C}: 8.2,18.1 ; 15^{\circ} \mathrm{C}: 8.4,18.8 ; 20^{\circ} \mathrm{C}: 11.0,23.0 ; 25^{\circ} \mathrm{C}: 13.2,27.1$, respectively) despite using a log function, the TRL4 stage-gate is past enough in principle to proceed. 
TRL5 - technology validated in relevant environment (industrially relevant environment in the case of key enabling technologies)

The UK test data set was confirmed to cover most of England (Figure 5(a)-(c)) and using the diagnostic ratios outlined by Griffiths (1979), mainly (but not exclusively) was comprised of the '[A]' population group (Figure 6 (c)). Approximate attribution to breeding group ['A'] or ['B'] in the TRL5 data set was by defining a 'cut' orthogonal to the common regression of setal ratios over the $\mathrm{T}$ and PT collections which prevented the Tyrophagus nieswanderi individuals being associated with the region of ['B']. The sampled locations broadly summarise the expected pattern of actual temperatures for the summer and winter in the UK (Figure 5 (d)-(e) respectively).

The environmental temperature predicted from the logistic equation on mite sizes does fall with latitude in winter as expected (and unsurprisingly not so in the summer) - Figure 7 validating this paper's approach in principle at TRL5. This method appears to be a sensitive approach to environmental measurement. These tyrophagid mites show a size difference over 6 degrees of latitude that Varroa jacobsoni does not show over a span of 15 degrees on the north-south axis (Grobov et al. 1979). Predictions and actual temperature data from that location are shown in Figure 8 (a) for females and males using the same above equations. Male data generally agrees with that of females suggesting that for the measures used in this study, male T.putrescentiae individuals are of the same fundamental design (but just different size) than females. Note that the UK mite sample does not cover the upper reaches of the published temperature limits for T.putrescentiae in the laboratory.

However, the logistic multiplex model predicted temperature does not in itself predict well that actual meteorological temperature of the location where the individual mite was collected (c.f. flat regression lines in Figure 8 (a)). Rather as can be seen in the Figure 8 (b), over both the summer (c.f. right-hand vertical cluster) and the winter (c.f. left-hand elongate vertical cluster) substantial variation in predicted temperature is found (agreeing with Figure 7). No clear distinction between field-collected mites attributed to the '[A]' versus '[B]' population groups were seen and similar variations were indicated for the small numbers 
of T.nieswanderi (results not shown). Exact species attribution appears unnecessary here. Comparing this to the range of predictions for laboratory data of an independent population of 36 female T.putrescentiae individuals kept at $15^{\circ} \mathrm{C}$ originally from Copra lying beneath trees in the Solomon Isles from the work of Owen 1978 (data not put into the public domain), shows the variation over locations at a nominal AvMax, to be far in excess of that expected from a uniform well-controlled environment. It is also bigger than the nominal approximately $10^{\circ} \mathrm{C}$ worst case range extracted above from Figure 4. Either the actual temperature at the sampled locations is markedly different from that estimated from the nearest Met. Office Historical stations or something extra in the wild is engendering a greater level of variation. Perhaps the assumption that mites in the wild behave like mites in a laboratory is not true. Unfortunately there were very few replicate samples of mites from the same location at the same date in order to examine if the standard deviations $(\sigma)$ of raw setal and body length measurements within date by location were similar to Table 2. Similarly by virtue of lack of this replication, the standard deviations of prediction could not be compared to those of Table 3 and Table 4

Whilst any over-warming in the wild might be explainable, there is no clear reason why mite-estimated temperatures at the sampled locations should have been markedly less than that expected meteorologically (i.e. how to produce large mites in the warm?). Assuming that measurement error is broadly constant and that a wild population is made up of differently responding individuals in the same way as that a laboratory population is, one is forced to consider something extra is physically or biologically different between sampled locations. For T.putrescentiae, there was no obvious relationship of this disparity between predicted and actual temperature with latitude nor with longitude over the summer and winter seasons (results not shown). Perhaps feeding conditions vary? Small mites need less energy to survive and therefore reduced size would be expected in locations with poor food quality conditions (recall small mites $\equiv$ warm predicted conditions). Unfortunately very little is known of the detailed conditions where the TRL5 samples were taken other than it is likely that food was ad libitum - locations included houses, farms, ships, animal buildings etc (none of which were labeled as refrigerated, nor was any cold night-air forced ventilation as used in 
stored product beetle pest control - Thorpe et al. 1982 - recorded). From Figure 8 (b), there appears to be no trend as one passes from summer to winter in the first instance, showing that there is little (if any) hidden degree of correlation overall with anything seasonal. However, in fact, re-expressing the data as standardised $z$-scores (Figure 9 (a)) and setting summer as a reference basis shows how in the winter possible local heating by, and of, the T.putrescentiae population may explain the confounding. This tendency with falling actual temperature is shown to be present even in the lower temperatures of the summer months (c.f. convex hull envelope and regression lines in the Figure 9 (b) which suggest a parabolic tendency). Tyrophagus putrescentiae would in a sense be regulating its environment to obviate the cold by clustering perhaps or seeking more insulated environments. Alternatively, recalling that the second minor component in the SVD above contrasted the length of seta L2 with the non-lateral measures, could the winter mites be somehow different shape than the summer mites (i.e. comparatively 'fatter' being less 'elongate' with greater L2 values in the cold?). That is, some sort of developmental response to prolonged temperature exposure? Could there be a seasonality in body mass (as in ants - Kaspari 2005) or in the body composition of T.putrescentiae? Plotting the 'logistic predicted value - monthly AvMax' i.e the residual of prediction versus $\log _{e}(L 2)$ gives a reasonably well fitting straight line $\left(R^{2}=0.51\right.$, result not shown). Linear application of such an adjustment to the overall model certainly renders the location-to-location variation more typical of the $10^{\circ} \mathrm{C}$ min-to-max prediction range (cf. Figure 4) but effectively removes any seasonality (i.e. the loss of signal in the summer in Figure 10. The conclusion then would be that somehow T.putrescentiae populations were modifying their environment to ensure equable temperature conditions all year round (through heating from potential population size or actively seeking of certain cooler microhabitats) and that this population-effect shift is indicated by indirectly producing mites of different levels of L2 growth. Astigmatid 'blooms' in stored material would then be almost like a honey bee colony, environmentally homeostatic over time with seasonal complements of 'summer bees' (aka smaller slightly more elongate 'summer mites' with proportionately smaller L2 setae) and 'winter bees' (aka larger slightly more stubby 'winter mites' with proportionately larger L2 setae)). Using unadjusted measurements of these would confound the workings of equation (1) and the accuracy of any mite-derived logistic predictions of temperature from equation (2). 
TRL5 in practice is shown but with some caveats that need follow-up work.

\section{TRL6 - technology demonstrated in relevant environment (industrially relevant environment in the case of key enabling technologies)}

Given the results at TRL5 and no further data being available to the author on the local micro-climate of the test data set specimens actually used, it is not yet possible to proceed further with this biotechnology. Next steps planned for the logistic equation methodology would have been the critical examination of the field-collected TRL5 Overseas specimens in the test data sets. Then examining the geographic variability over different countries of tyrophagid response to temperature. The Overseas sample came from hotter climes than the UK and would have been a further validatory test of the method.

Accordingly, no assertions for the museum categorisations of undated or unknown geography specimens can yet be made.

\section{Discussion}

TRL3 and TRL4 stage-gates were passed in developing this biotechnological proposition. The single important eigenvector indicates that in general, setae and body length undergo the same temperature response processes just at different scales. TRL5 was partially achieved for Tyrophagus putrescentiae. There is an utility of using an acarid mite multiplex assay in picking up different features of environmentally-driven variation. However there are limitations to this study as it stands.

Firstly, there is the adequacy of the logistic regression as a prediction equation. Herein this sigmoid is described by indicative draft parameters, with no claim being made as to the diagnostic use of these actual values. Also recall that measures were $\log _{e}$ transformed at the outset which compresses actual variation scales. Independent laboratory manipulations need to be carried out to replicate this pilot before advancing any particular technology. There is 
also the apparent considerable individual mite-to-mite variation observed (even in laboratory cultures). This may preclude Tyrophagus putrescentiae being a highly sensitive indicator of temperature in the wild where other confounding factors on top of this would be expected. Suggested improvements include using 5 and $30^{\circ} \mathrm{C}$ samples to get the tail behaviour in any re-run of laboratory work. Using a power or asymmetric logistic function (e.g. Nelder 1961. 1962) can change the tail behaviour and would impact TRL3 too. Shepherd (1982) lists that at $90 \% \mathrm{RH}$ there was no development at $5^{\circ} \mathrm{C}\left(10^{\circ} \mathrm{C}\right.$ being the lowest tested in depth), and though development times are of the order of those at $25^{\circ} \mathrm{C}$ - for T.putrescentiae exposed to $35^{\circ} \mathrm{C}$, there was very high juvenile mortality at $35^{\circ} \mathrm{C}$. Other acarids also appear to be able to sustain colder temperatures more than extreme heat conditions (Cunnington|1984). By what mechanism this heat death occurs in mites is not clear (Madge 1965). Abbar (2016) explores time-mortality relationships for T.putrescentiae specifically. Perhaps long time exposure to higher temperatures in conditions of high moisture would mean tyrophagid mites habituate and could live longer and thus the $36^{\circ} \mathrm{C}$ cut-off be posed higher at TRL4. From above, genetic adjustment or at least the degree of inbreeding might be of relevance (the latter could be assessed in follow-up work by new technologies such as Next Generation Sequencing). More laboratory investigations on thermal tolerance, habituation and size adaptations in tyrophagids would inform progress.

Secondly, many factors, of course, feed into the final size (and shape) of a poikilotherm - their fundamental genetics (including any inbreeding - Trotta et al. 2011) as well as their environmental exposures (Pakkasmaa (2001); Costa et al. 2010). In frogs (Vogt and Jameson 1970), the rapidity of response of body sizes with temperature may indicate initially some of the variation is non-genetic (as assumed by this paper's postulate) followed later by periods of genetic stabilisation. Krzeczkowski (1962) shows for Tyrophagus noxius (= T. putrescentiae), not only that higher temperatures produce smaller female mites in particular (although both sexes do change), but that higher relative humidity engenders a greater hydration and hence a greater size in their total hysterosoma estimated from living specimens. Food quality was also important as was the dwarfing consequences of T.noxius (= T. putrescentiae) living at high densities. High densities yielded an apparent persistent 
dwarfing effect. Inbreeding can affect quantitative features in astigmatids (Wolska 1980) the original $20^{\circ} \mathrm{C}$ laboratory cultures used by Shepherd 1982 must have been highly inbred before use (T13 for at least a decade - Table 1). Rhizoglyphus robini (Acaridae) is known to show fluctuating asymmetry (Radwan et al. 2003) which would compromise setal length accuracies if repeated in tyrophagids. Any of these biases could in theory feed through into an inaccurate prediction of environmental temperature from a museum specimen's measurements. However, the T.putrescentiae 'thermometer' using discrete fixed chitinous structures (IL, D1, D2, L2) as its basis at least negates any issues of variable relative humidity directly affecting the visual act of taking the measurements underlying the predictions. It is assumed that in the wild for most free-living astigmatids food is unlikely to be limiting in the first instance on arrival at the ephemeral patch that they find themselves in. So changes in size in field collected individual specimens due to reduced nutritional status is not taken to be the first cause of any confounding. After all the time for an individual mite to grow and develop is much smaller than that for the population to grow to huge numbers and impact the quality of food.

Rather it is interpreted that what the size changes in T.putrescentiae indicate in this study herein is the local ambient (to the mite) not the global ambient (for the location) environmental temperature. Usually in the wild, mites prefer micro-habitats that have substantial local heating, i.e. decomposing organic matter, where fungi and bacteria may be also active. The variation seen in both the summer and winter collection periods in predicted environmental temperature from the mites' sizes compared to the estimated global temperature for that location (Figure 8 (b)) does suggest 'hot-spots' of higher temperatures experienced for some of those mites collected. This appears more frequent over places in the colder winter season than the warmer summer (Figure 9 (a)). High densities of mites would induce metabolically driven hot-spots in any semi-insulated conditions, which thus if persistent over time in turn engender smaller 'dwarfed' mites (and thus a higher logistic equation predicted temperature). Microbial activity in the damper winter months may exacerbate this heating effect. In the warmer summer, mites would be naturally smaller and could not get drastically smaller courtesy of further local heating unlike the larger mites 
in the colder winter global ambient temperatures (Figure 9 (b)). The corollary here is that one might expect higher densities of infestations in the winter therefore (and perhaps less attendant microbial activity in the drier summers). Whether this population density is actively regulated behaviourally by clustering of individuals (perhaps pheremonally mediated) awaits further work. So, in this way the actual life strategy of T.putrescentiae (Mueller et.al. 2006) may obviate choosing it as the 'self-thermometer' for museum specimens. Perhaps a more solitary mite where density-related effects should not occur would be a better choice? One that was more exposed to the global ambient temperature for that location? One that by its very existence could not gregariously generate local heat from any high densities or semi-enclosed crypsis?

TRL6 for T.putrescentiae over the UK could only be attempted given detailed data-logging of micro-temperatures in the field repeatedly at locations systematically geographically dispersed, together with the targeted collection and characterisation of mites at the same locations and measures of their local density over time. Density could be assessed using standard volume or weight samplings plus the quantitative flotation method of Thind (2000) (as offered by https://www.fera.co.uk/crop-health/insect-monitoring - for more details see FERA 2019). Body composition could also then be checked over time. One then might also be able to explain apparent somewhat 'cold' temperatures indicated by certain mite samples in what should be warm summers. Then perhaps there could be found a predictive relationship of mite size with the global ambient temperature of the mite location (without confounding heating effects) over the seasons. Through this, an assessment of mite form, and the probability of heating effects (and at what scale they occur) would be possible, so a stochastic deconvolution could be done using mite measurements to yield a Bayesian probability interval around any inferred temperatures from field collected specimens allowing for likely population densities (perhaps also informed by any subtle changes in shape and L2 values). Until this is done, expansion of this currently TRL5 method to international samples over a wider range of latitude and longitude (TRL7 - system prototype demonstration in operational environment) is not prudent. 
This paper simply illustrates what might be usefully done to build upon an existing body of acarine prior art. I call upon other acarologists to check if this can be operationalised better with other mites in other biome settings (Remmert 1981). Furthermore to consider how TRL8 (system complete and qualified) or TRL9 (actual system proven in operational environment, competitive manufacturing in the case of key enabling technologies; or in space) for any environmental assessment using world-wide museum specimens might ever be judged.

This general approach is commended to acarologists to challenge, consider improving and deploying.

\section{Acknowledgement(s)}

All new data generated and analysed by the author during this study at the Oxford Centre for Industrial and Applied Mathematics plus any model specifications are included in this published article - or in compliance with EPSRC's open access initiative the TRL5 'all-complete' dataset of Tyrophagus putrescentiae individuals is available from https: //doi.org/10.5287/bodleian:0005Y00Y0, Thanks go to to the generosity of:- the late Don Griffiths for access to the dataset from Shepherd (1982); Barry OConnor, University of Michigan for repeated help with astigmatid classification; Charley Eiseman and Pavel Klimov for permission to reuse their colour photographs. Above all, I thank my wife Diane for supporting my involvement in this field over many years. The author is a Royal Society Industrial Fellow (IF110047) at the Mathematical Institute, University of Oxford.

\section{Disclosure statement}

The author declares that they have no known conflicts of interest. No competing claims are known. This article does not contain any studies with human participants or vertebrate animals performed by any of the authors. 


\section{Funding}

Self-funded study.

\section{Open access}

This article is distributed under the terms of the Creative Commons Attribution 4.0 International License (http://creativecommons.org/licenses/by/4.0/). This permits unrestricted use, distribution, and reproduction in any medium, provided you give appropriate credit to the original author(s) and the source, provide a link to the Creative Commons license, and indicate if changes were made.

\section{References}

Abbar S (2016) Methods for management of Tyrophagus putrescentiae (Schrank) (Sarcoptiformes: Acaridae) in dry-cured ham facilities. Dissertation submitted in partial fulfilment of the requirements for the degree Doctor of Philosophy, Department of Entomology, College of Agriculture, Kansas State University, Manhattan, Kansas, USA 132pp

Aguilar-Medrano R, Frédérich B, de Luna E, Balart E (2011) Patterns of morphological evolution of the cephalic region in damselfishes (Perciformes: Pomacentridae) of the Eastern Pacific. Biological Journal of the Linnean Society 102:593-613

Atkinson.D (1994) Temperature and organism size: a biological law for ectotherms? Advances in ecological research 25:1-58

Bakker FT, Antonelli A, Clarke JA, Cook JA, Edwards SV, Ericson PGP, Faurby S, Ferrand N, Gelang M, Gille- spie RG, Irestedt M, Lundin K, Larsson E, Matos-Marav P, Mller J, von Proschwitz T, Roderick GK, Schliep A, Wahlberg N, Wiedenhoeft J, Kllersj M. (2020). The Global Museum: natural history collections and the future of evolutionary science and public education. PeerJ 8:e8225 http://doi.org/10.7717/peerj.8225

Barlow GW (1961) Causes and Significance of Morphological Variation in Fishes. Systematic Biology 10(3): 105117

Bowman CE (2020) Is there an advantage in Astigmatid chelicerae? Experimental and Applied Acarology (submitted)

Costa C, Vandeputte M, Antonucci F, Boglione C, Menesatti P, Cenadelli S, Parati K, Chavanne H, 
Chatain B (2010) Genetic and environmental influences on shape variation in the European sea bass (Dicentrarchus labrax). Biological Journal of Linnean Society 101:427-436

Cunnington AM (1984) Resistance of the grain mite Acarus siro L. (Acarina, Acaridae) to unfavourable physical conditions beyond the limits of its development. Agriculture, Ecosystems and Environment $11: 319-.339$

Dosch JJ (2007) On dead birds' tales: Museum specimen feathers as historical archives of environmental pollutants. Environmental History 12(3):661-665

EU Commission (2014) Technology readiness levels (TRL). HORIZON 2020 WORK PROGRAMME 2014-2015 General Annexes: Extract from Part 19 - Commission Decision C(2014)4995G. https://ec.europa.eu/research/participants/data/ref/ h2020/wp/2014_2015/annexes/h2020-wp1415-annex-g-trl_en.pdf

FERA (2019) You mite miss it. Professional Pest Controller Magazine. 95:22-24

Griffiths DA (1979) The morpho-species and its relationship to the biological species in the genus Tyrophagus (Acaridae, Acarina). Recent Advances in Acarology 1:199-212

Griffiths DA, Atyeo WT, Norton RA and Lynch CA (1990) The idiosomal chaetotaxy of astigmatid mites. Journal of Zoology 220(1):1-32

Grobov OF, Pulenetz NM, Sofronova SL (1979) Geographically determined variability in the size of the dorsal plate of females of Varroa jacobsoni Oudemans. 27th International Bee Breeding Conference, Athens, Greece. Apimondia 379-383 (in German)

Houck MA, OConnor BM (1966) Temperature and host effects on key morphological characters of Hemisarcoptes cooremani and Hemisarcoptes malus (Acari: Hemisarcoptidae). Experimental and Applied Acarology 20(12):667-682 doi:10.1007/BF00051552

Kaspari M (2005) Global energy gradients and size in colonial organisms: Worker mass and worker number in ant colonies. Proceedings of the National Academy of Sciences 102(14):5079-5083 https://www.pnas.org/cgi/doi/10.1073/pnas.0407827102

Kingsolver JG, Huey RB (2008) Size, temperature, and fitness: three rules. Evolutionary Ecology Research 10: 251-268

Klimov PB, Lekveishvili M, Dowling APG, OConnor BM (2004) Multivariate analysis of morphological varaition in two cryptic species of Sancassania (Acari: Acaridae) from Costa Rica. Annals of the Entomological Society of America 97(2):322-345

Krzeczkowski K (1962) Influence of certain ecological factors on the body size of adult Tyrophagus noxius Zachw. (Tyroglyphidae, Acarina). Prace Naukowe Instytutu Ochrony Roślin 4(1):155-176 (in Polish) 
Lynch CA (1989) Two new species of the genus Tyrophagus (Acari: Acaridae). Journal of Zoology, London 219:545-567

Madge.DS (1965) The effects of lethal temperatures on oribatid mites. Acarologia 7(1):121-130

Bryan S. McLean, Kayce C. Bell, Jonathan L. Dunnum, Bethany Abrahamson, Jocelyn P. Colella, Eleanor R. Deardorff, Jessica A. Weber, Amanda K. Jones, Fernando Salazar-Miralles, Joseph A. Cook(2016) Natural history collections-based research: progress, promise, and best practices. Journal of Mammalogy 97(1):287-297 https: //doi .org/10.1093/ jmammal/gyv178

Mueller DK, Kelley PJ, VanRyckeghem AR (2006) Mold mites Tyrophagus putrescentiae (Shrank) in stored products. 9th International Working Conference on Stored Product Protection. PS9-7-6283. Psocids, Mites, and Other Contaminants p1117-1122

Nelder JA (1961) The fitting of a generalization of the logistic curve. Biometrics 17(1):89-110

Nelder JA (1962) Note: An alternative form of a generalised logistic equation. Biometrics 18(4):614616

Owen DA (1978) Setal length in the genus Tyrophagus (Acaridae, Acarina) as a measure of intra and interspecific variability. Department of Biology, Industrial Student Report, Liverpool Polytechnic, UK $27 \mathrm{pp}$

Pakkasmaa S (2001) Morphological differentiation among local trout (Salmo trutta) populations. Biological Journal of the Linnean Society 72:231-239

Radwan J, Watson PJ, Fraslow J, Thornhill R (2003) Procrustean analysis of fluctuating asymmetry in the bulb mite Rhizoglyphus robini Claparede (Astigmata: Acaridae). Biological Journal of the Linnean Society 80:499-505

Remmert H (1981) Body size of terrestrial arthropods and biomass of their populations in relation to the abiotic parameters of their milieu. Oecologia 50:1213

Sánchez-Ramos I, Castalñera P (2001) Development and survival of Tyrophagus putrescentiae (Acari: Acaridae) at constant temperatures. Environmental Entomology 30(6):1082-1089

Schmitt CJ, Cook JA, Zamudio KR, Edwards SV. (2018) Museum specimens of terrestrial vertebrates are sensitive indicators of environmental change in the Anthropocene. Philosphical Transactions of Royal Society B 374:20170387 http://dx.doi.org/10.1098/rstb.2017.0387

Seeman OD, Nahrung HF (2018) In short- or long-term relationships, size does matter: body size patterns in the Mesostigmata (Acari:Parasitiformes). International Journal of Acarology 44(8) https://doi.org/10.1080/01647954.2018.1530299

Shepherd IR (1982) An investigation of setal and idiosomal lengths as a basis for subspecific division in the mite Tyrophagus putrescentiae, and, An investigation into reproductive isolation mechanisms 
in mutually incompatible populations of the mite Tyrophagus palmarum. Department of Biology, Industrial Student Report, Liverpool Polytechnic, UK 94pp

Suarez AV, Tsutsui ND (2004) The value of museum collections for research and society. BioScience 54(1):66-74

Thind BB (2000) Determination of low levels of mite and insect contaminants in food and feedstuffs by a modified flotation method. Journal of Association of Official Analytical Chemists 83(1):113-119

Thorpe GR, Cuff WR, Longstaff BC (1982) Control of Sitophilus oryzae infestation of stored wheat: An ecosystem model of the use of aeration. Ecological Modelling 15:331-351

Trotta V, Cavicchi S, Guerra D, Andersen DH, Babbit GA, Kristensen TN, Pedersen KS, Loeschcke V, Pertoldi C (2011) Allometric and non-allometric consequences of inbreeding on Drosophila melanogaster wings. Biological Journal of the Linnean Society 102:626-634

Vogt T, Jameson DL (1970) Chronological correlation between change in weather and change in morphology of the Pacific Tree Frog in Southern California. Copeia (1):135-144

Wolska KJ (1980) Effect of inbreeding on quantitative features of copra mite, Tyrophagus putrescentiae Schr., Acarina: Acaridae. Genetica Polonica 21(3):291-308 
Table 1. Details of origin of Tyrophagus putrescentiae 'stock' laboratory cultures (maintained at $20^{\circ} \mathrm{C}$ ) used by Shepherd (1982). Capital letters in square brackets [...] represent isolated breeding groups - Griffiths (1979).

\begin{tabular}{llll}
\hline Culture & Origin & Where Found & Date \\
\hline T2 ['B'] & Berks, UK & Chocolate bar & 1 Nov 1967 \\
T13 ['A'] & South Africa & Groundnuts & 24 Jul 1968 \\
T58 ['A'] & Surrey, UK & $\begin{array}{l}\text { Ceratocystis ulmi, in galleries } \\
\text { of the Elm bark beetle }\end{array}$ & 3 Dec 1976 \\
\hline
\end{tabular}


Table 2. Summary in $\mu \mathrm{m}$ of dataset from Shepherd (1982). Mean $(\mu)$ and Standard deviation $(\sigma)$ for female adult Tyrophagus putrescentiae training data set female individuals $(N=450)$ and validation data set $(N=600)$ by population (each populationtemperature combination $N=50$ ). Validation data $=$ reduced gene pool female adults from egg founders ex original laboratory cultures exposed to that new temperature regimen. $*=$ Closest representative sample to original 'stock' maintenance cultures.

\begin{tabular}{|c|c|c|c|c|c|c|c|c|c|}
\hline \multirow[t]{2}{*}{ Population } & \multirow{2}{*}{$\begin{array}{l}\text { Temp- } \\
\text { erature } \\
{ }^{\circ} \mathrm{C}\end{array}$} & \multirow{2}{*}{$\begin{array}{l}\mathrm{IL} \\
\mu\end{array}$} & \multicolumn{3}{|c|}{ D1 } & \multirow{2}{*}{$\begin{array}{c}\mathrm{D} 2 \\
\mu\end{array}$} & \multicolumn{3}{|c|}{$\mathrm{L} 2$} \\
\hline & & & $\sigma$ & $\mu$ & $\sigma$ & & $\sigma$ & $\mu$ & $\sigma$ \\
\hline \multicolumn{10}{|l|}{ Training } \\
\hline \multirow[t]{3}{*}{$\mathrm{T} 2$} & 10 & 208.3 & 17.3 & 43.5 & 7.2 & 78.5 & 11.0 & 34.7 & 5.4 \\
\hline & 15 & 218.3 & 14.3 & 39.1 & 4.3 & 77.3 & 8.1 & 31.0 & 2.7 \\
\hline & 25 & 185.5 & 10.3 & 31.7 & 3.2 & 64.2 & 7.6 & 25.7 & 3.8 \\
\hline \multirow[t]{3}{*}{ T13 } & 10 & 232.8 & 17.6 & 39.1 & 4.8 & 102.9 & 12.7 & 45.7 & 5.3 \\
\hline & 15 & 216.1 & 11.0 & 34.7 & 3.9 & 101.0 & 9.8 & 36.0 & 4.5 \\
\hline & 25 & 203.1 & 12.7 & 30.2 & 2.8 & 92.1 & 8.4 & 35.1 & 4.1 \\
\hline \multirow[t]{3}{*}{ T58 } & 10 & 234.3 & 15.2 & 34.9 & 4.5 & 119.0 & 13.6 & 45.7 & 5.1 \\
\hline & 15 & 210.6 & 10.6 & 30.6 & 2.7 & 110.8 & 12.9 & 36.3 & 3.4 \\
\hline & 25 & 192.4 & 10.4 & 26.4 & 2.6 & 94.4 & 6.3 & 28.7 & 2.2 \\
\hline \multicolumn{10}{|l|}{ Validation } \\
\hline \multirow[t]{4}{*}{ PT2 } & 10 & 234.9 & 13.6 & 48.0 & 5.3 & 95.5 & 11.7 & 36.1 & 4.4 \\
\hline & 15 & 219.5 & 11.8 & 42.6 & 4.9 & 91.6 & 8.9 & 35.5 & 3.1 \\
\hline & $20 *$ & 206.4 & 13.1 & 39.9 & 3.4 & 88.3 & 9.1 & 32.2 & 3.0 \\
\hline & 25 & 184.4 & 7.1 & 33.8 & 3.8 & 72.2 & 6.4 & 26.4 & 2.8 \\
\hline \multirow[t]{4}{*}{ PT13 } & 10 & 239.6 & 11.3 & 39.5 & 4.3 & 108.8 & 10.1 & 46.4 & 6.7 \\
\hline & 15 & 237.4 & 16.0 & 37.7 & 3.5 & 107.4 & 10.4 & 44.6 & 4.1 \\
\hline & $20 *$ & 221.1 & 18.9 & 33.2 & 8.5 & 95.0 & 8.5 & 40.0 & 4.3 \\
\hline & 25 & 200.6 & 9.1 & 31.0 & 2.2 & 92.2 & 5.8 & 35.7 & 3.0 \\
\hline \multirow[t]{4}{*}{ PT58 } & 10 & 225.0 & 14.0 & 33.9 & 3.4 & 112.3 & 12.4 & 39.8 & 4.8 \\
\hline & 15 & 211.4 & 16.4 & 32.1 & 2.6 & 112.7 & 8.4 & 37.0 & 3.5 \\
\hline & $20 *$ & 196.1 & 8.3 & 29.4 & 2.4 & 99.1 & 8.6 & 32.0 & 3.0 \\
\hline & 25 & 177.4 & 9.5 & 26.1 & 2.1 & 82.0 & 6.2 & 27.5 & 2.6 \\
\hline
\end{tabular}


Table 3. TRL3 Eigen-analysis of average within-culture within-temperature covariance matrix across $\log _{e}$ measures in augmented training data set $(N=750)$ of $T y$ rophagus putrescentiae female mites in order to form surrogate $\Theta(1$. Note essentially only one component explains sample variation ( $86 \%$; rest is spherical noise) and that the allometric coefficient for setal length versus IL is around 0.3 .

\begin{tabular}{ccccc}
\hline Item & Component1 & Component2 & Component3 & Component4 \\
\hline Eigenvalues & 0.024227369 & 0.007050308 & 0.005832541 & 0.003322981 \\
& & & & \\
IL & -0.1804933 & -0.1196814 & 0.06890973 & 0.9738326208 \\
D1 & -0.5726658 & -0.4548077 & -0.67238666 & -0.1144553869 \\
D2 & -0.5586939 & -0.3323566 & 0.73406474 & -0.1963393142 \\
L2 & -0.5721338 & 0.8175362 & -0.06554831 & -0.0009298738 \\
\hline
\end{tabular}


Table 4. TRL3 Results (Mean and Standard deviation) from using overall prediction equation (2) derived surrogate $\Theta$ (1) from training data set Tyrophagus putrescentiae female individuals $(N=450)$ applied back upon themselves by population (each population-temperature combination $N=50$ ). See Figure 3

\begin{tabular}{|c|c|c|c|c|}
\hline Population & $\begin{array}{l}\text { Temperature } \\
\text { (actual) }\end{array}$ & $\begin{array}{c}\text { Logistic } \\
\text { Prediction }{ }^{\circ} \mathrm{C} \\
\mu\end{array}$ & $\sigma$ & $\begin{array}{c}\text { Error } \\
\%\end{array}$ \\
\hline $\mathrm{T} 2$ & $\begin{array}{l}10 \\
15 \\
25\end{array}$ & $\begin{array}{l}15.50 \\
17.13 \\
23.42\end{array}$ & $\begin{array}{l}3.81 \\
2.53 \\
2.86\end{array}$ & $\begin{array}{l}55 \\
14 \\
-6\end{array}$ \\
\hline T13 & $\begin{array}{l}10 \\
15 \\
25\end{array}$ & $\begin{array}{l}11.92 \\
14.60 \\
16.98\end{array}$ & $\begin{array}{l}1.84 \\
2.54 \\
2.36\end{array}$ & $\begin{array}{l}19 \\
-3 \\
-32\end{array}$ \\
\hline T58 & $\begin{array}{l}10 \\
15 \\
25\end{array}$ & $\begin{array}{l}11.92 \\
14.84 \\
20.15\end{array}$ & $\begin{array}{l}1.84 \\
2.29 \\
1.94\end{array}$ & $\begin{array}{l}19 \\
-1 \\
-19\end{array}$ \\
\hline
\end{tabular}


Table 5. TRL4 Results (Mean and Standard deviation) from using overall prediction equation (2) derived surrogate $\Theta$ (1) from training data set Tyrophagus putrescentiae female individuals $(N=450)$ applied upon validation data set females $(N=600)$ by population (each population-temperature combination $N=$ $50)$.

\begin{tabular}{ccccc}
\hline Population & $\begin{array}{c}\text { Temperature } \\
\text { (actual) }\end{array}$ & $\begin{array}{c}\text { Logistic } \\
\text { Prediction } \\
\mu\end{array}$ & \multicolumn{1}{c}{$\mathrm{C}$} & $\begin{array}{c}\text { Error } \\
\%\end{array}$ \\
\hline PT2 & 10 & 12.41 & 2.19 & 24 \\
& 15 & 13.74 & 1.92 & -8 \\
& 20 & 15.51 & 2.04 & -22 \\
PT13 & 25 & 21.33 & 2.60 & 15 \\
& 10 & 11.16 & 1.57 & 12 \\
& 15 & 11.68 & 1.37 & -22 \\
& 20 & 14.44 & 1.84 & -28 \\
PT58 & 25 & 16.46 & 1.59 & -34 \\
& 10 & & & \\
& 15 & 13.09 & 1.93 & 31 \\
& 20 & 14.05 & 1.50 & -6 \\
& 25 & 17.47 & 2.03 & -13 \\
& & 22.35 & 2.09 & -11 \\
\hline
\end{tabular}




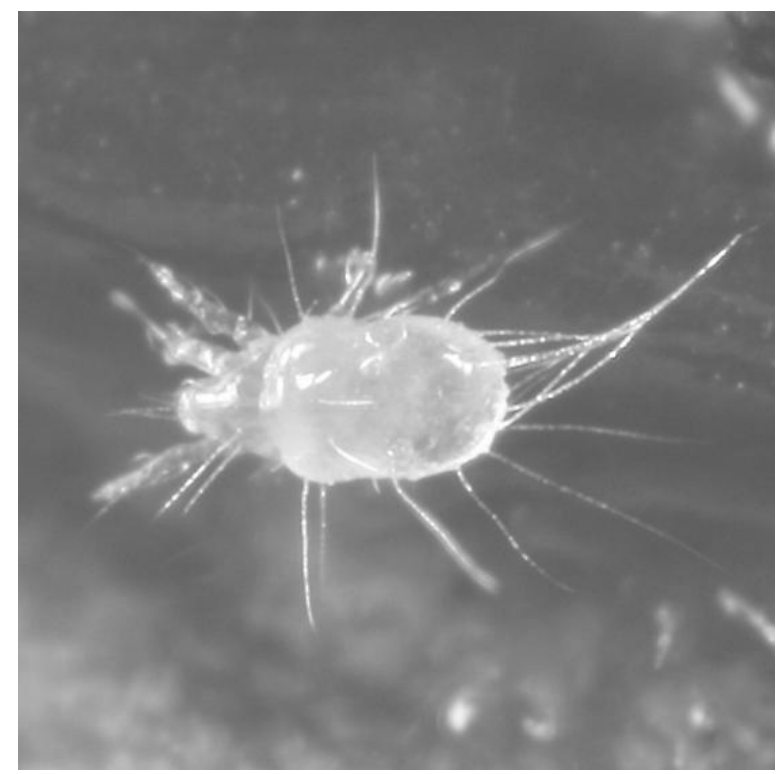

(a) Tyrophagus sp. in a tortricid moth (probably Episimus argutanus) witch-hazel (Hamamelis virginiana) leaf-roll, Pelham, Hampshire County, Massachusetts, USA, August 3, 2013 @ 2013 Charley Eiseman with permission.

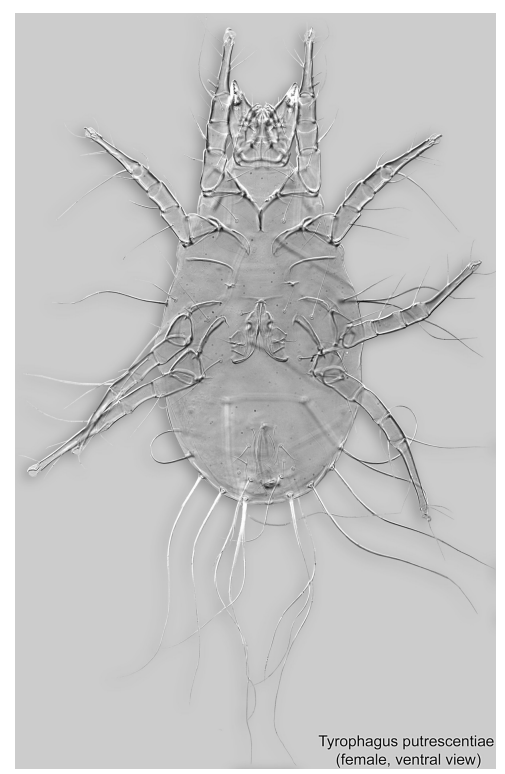

(b) Tyrophagus putrescentiae Female. Photo by Pavel Klimov, Bee Mite ID (idtools. org/id/mites/beemites) with permission.

Figure 1. Example acarid astigmatids. 

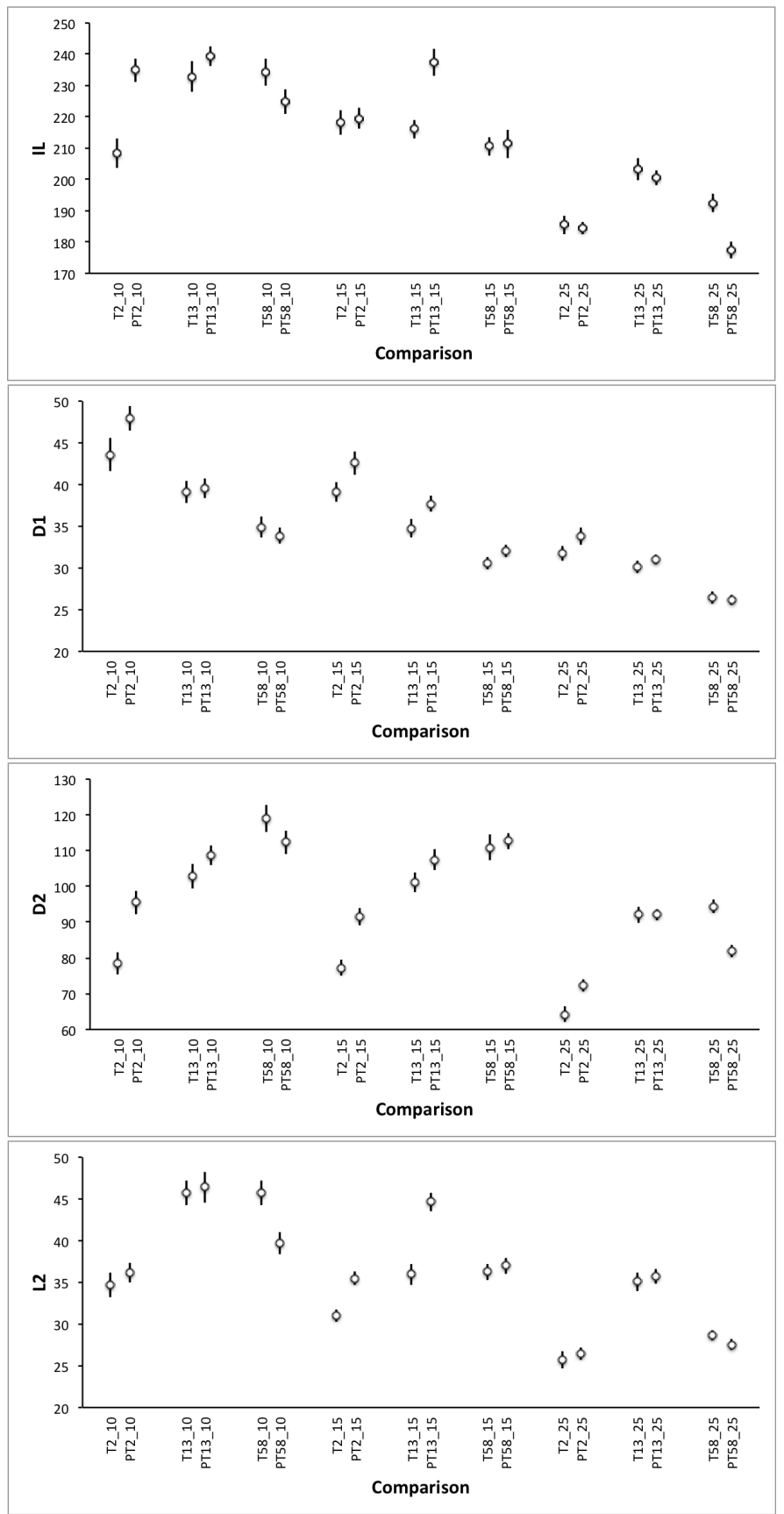

Figure 2. Mean (open circles) plus $95 \%$ confidence interval (black lines) for comparison between training and validation data Tyrophagus putrescentiae female sets segmented by population and temperature. Order from top to bottom : Idiosomal index IL, setal length D1, D2, L2. Measures in $\mu \mathrm{m}$. Overlap of lines within pair indicates no difference. No systematic trends are clear apart from an effect of temperature on mite scale. 


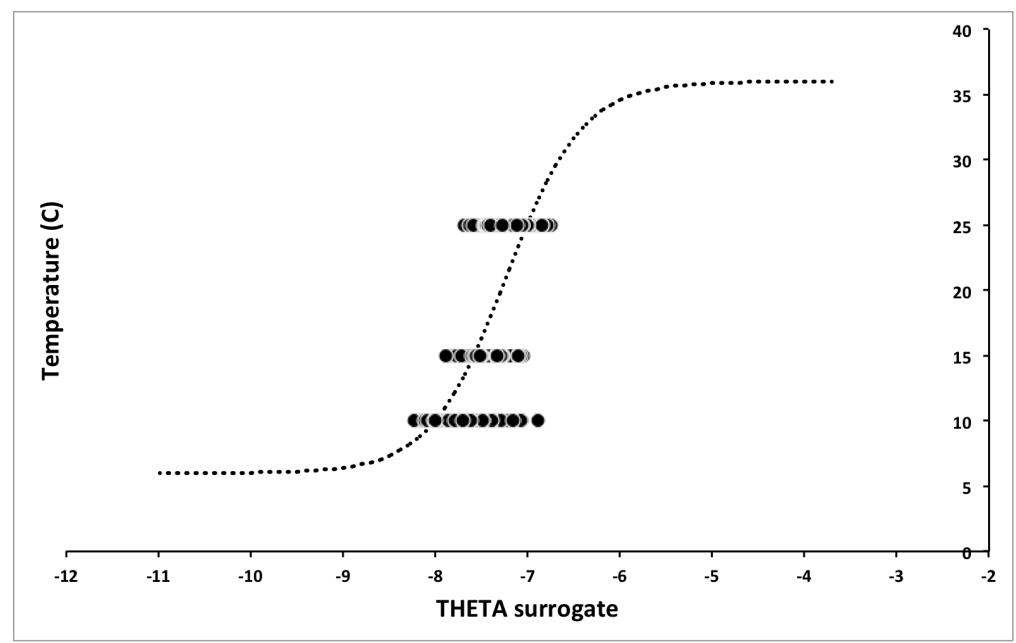

Figure 3. TRL3 Fitted sigmoid curve (dotted line) to $\Theta$ surrogate (1) from training data set Tyrophagus putrescentiae female individuals (T2, T13, T58 at $10,15,25^{\circ} \mathrm{C}$ as black circles) using equation 22, $\hat{b}=2.43014$ and $\hat{a}=17.57613$. See Table 3 Large mites are to the left, small mites to the right. 


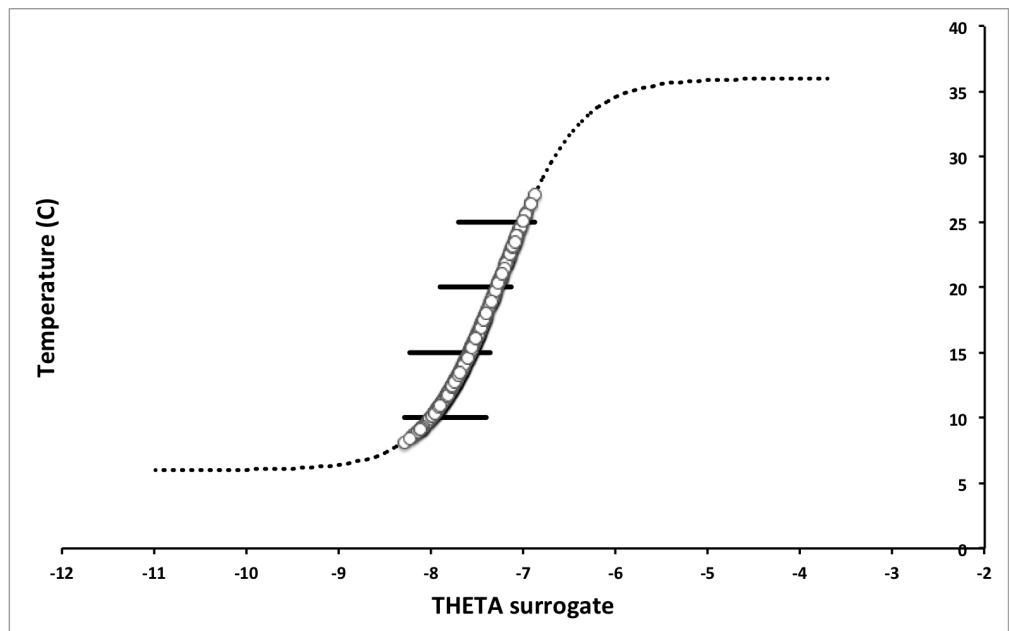

Figure 4. TRL4 Validation data set Tyrophagus putrescentiae female individuals (white circles) predicted as per fitted sigmoid curve (dotted line) in Figure 3 See Table 5 Black lines covers the range of mites PT2, PT13, PT58 at 10, 15, $20,25^{\circ} \mathrm{C}$. Large mites are to the left, small mites to the right. Note consilience with TRL3 (Figure 3 ) and continued coverage of the central almost linear section. 


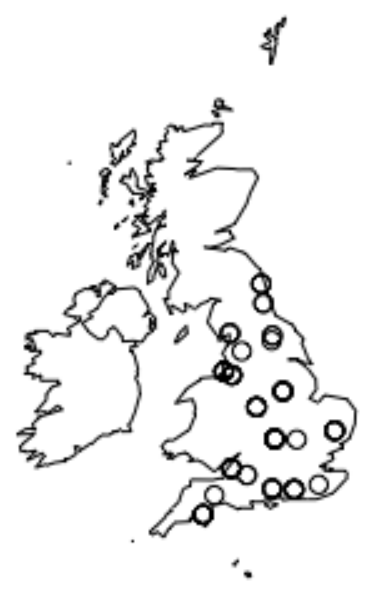

(a) Over all seasons.

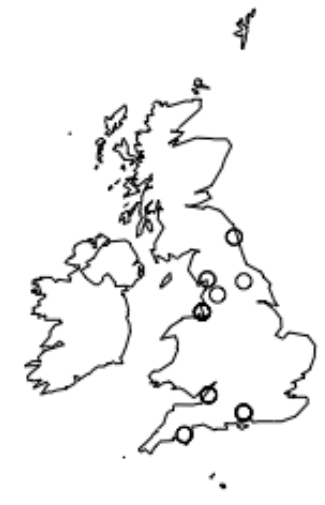

(b) Summer period (April - September inclusive).

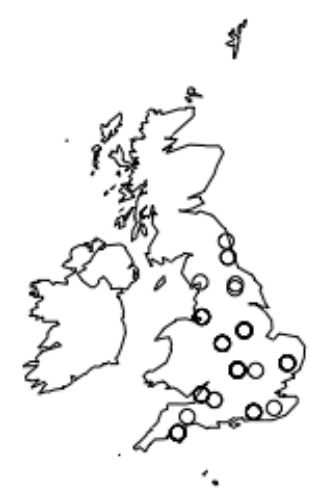

(c) Winter period (October - March inclusive).

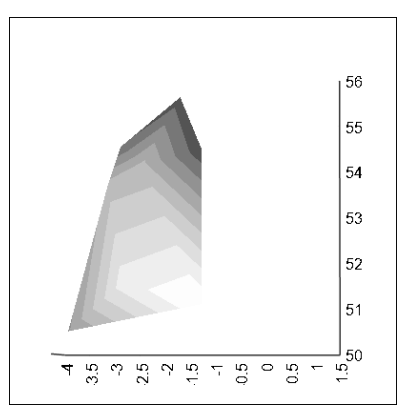

(d) Heat-map of Summer monthly AvMax from meteorological Historic Stations surrounding test dataset mite locations (dark $=$ colder, white $=$ warmer, $\mathrm{x}$-axis $=$ places sampled were warm in cerlongitude, $\mathrm{y}$-axis = latitude). Note tain winters. Higher latitude = cold north and warmer south.

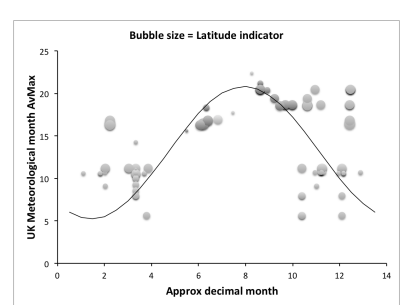

(e) UK Met Office Historic Station data across years showing general sinusoidal rise and fall in monthly tain winters
larger bubble. AvMax over locations. Note some

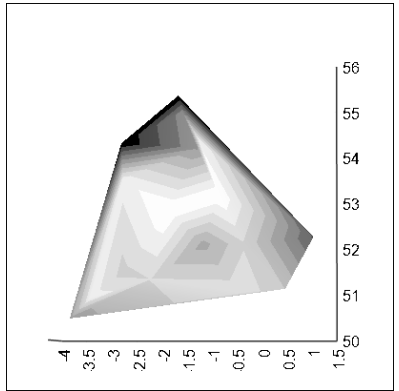

(f) Heat-map of Winter monthly AvMax from meteorological Historic Stations surrounding test dataset mite locations (dark = colder, white $=$ warmer, $\mathrm{x}$-axis $=$ longitude, $y$-axis = latitude). Note cold to north and central region of relative warmth.

Figure 5. Locations in UK of TRL5 Test dataset Tyrophagus putrescentiae individuals (males and females) over various years of collection with heatmap of observed temperatures from Met. Office. 


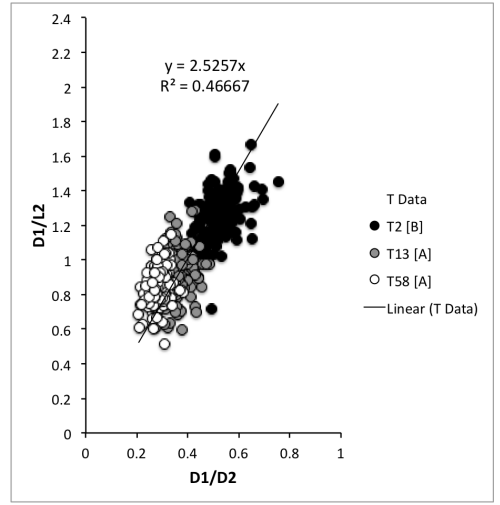

(a) TRL3 Training data set females.

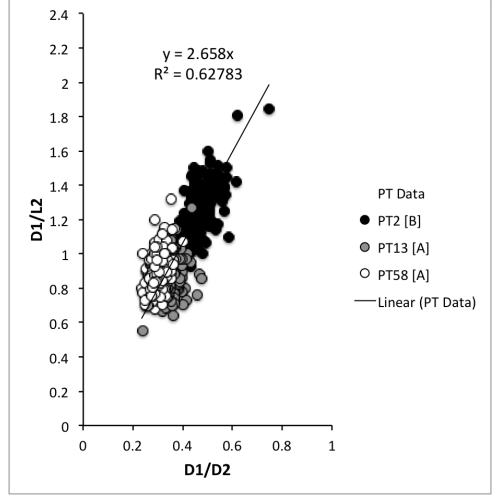

(b) TRL4 Validation data set females.

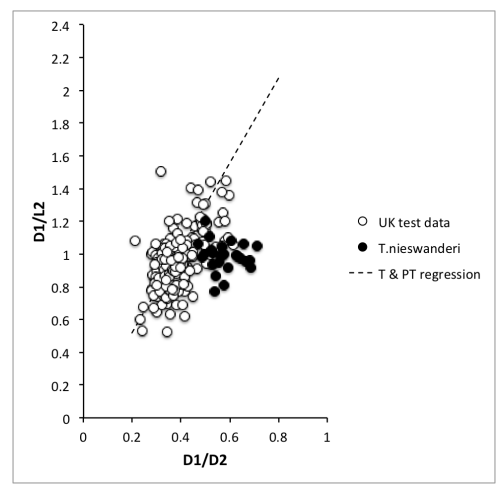

(c) TRL5 UK Test dataset T.putrescentiae mites (male and female) plus UK and overseas T.nieswanderi males and females.

Figure 6. Setal diagnostic ratios D1/L2 versus D2/L2 showing Tyrophagus putrescentiae population groups as per Griffiths (1979). Note separation but consistent trend over populations of breeding types. 


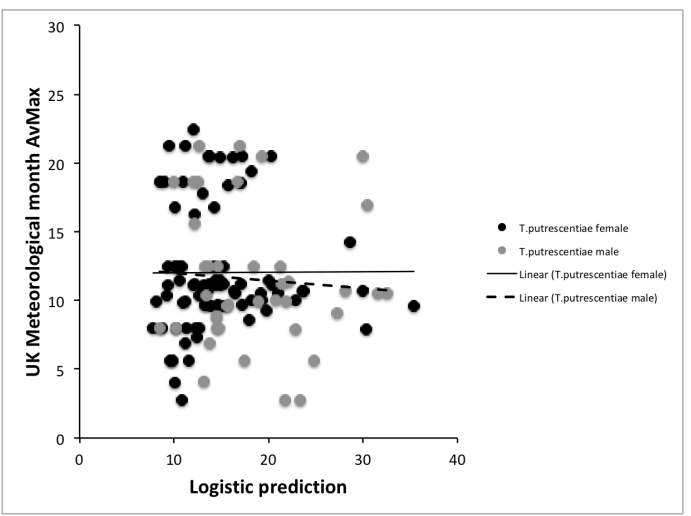

(a) Males and females behave similarly showing essentially no dependance between the $y$-axis upon the $x$-axis.

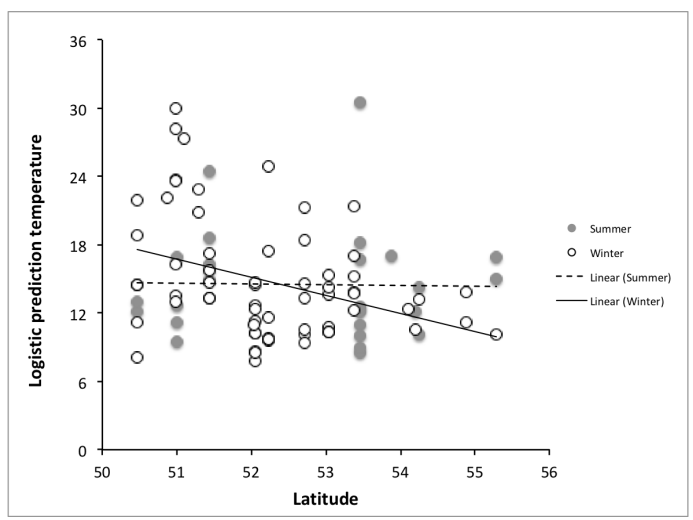

(b) Male and female logistic equation predicted temperature versus latitude of mite sample. In the winter (open circles), as one moves North the predicted temperature from mite size falls as expected. Little appears to change in the summer (grey circles). Vertical spread indicates that local temperature may confound the general actual relationship of mite size with global ambient temperature.

Figure 7. TRL5 UK Test dataset mites (Tyrophagus putrescentiae). 


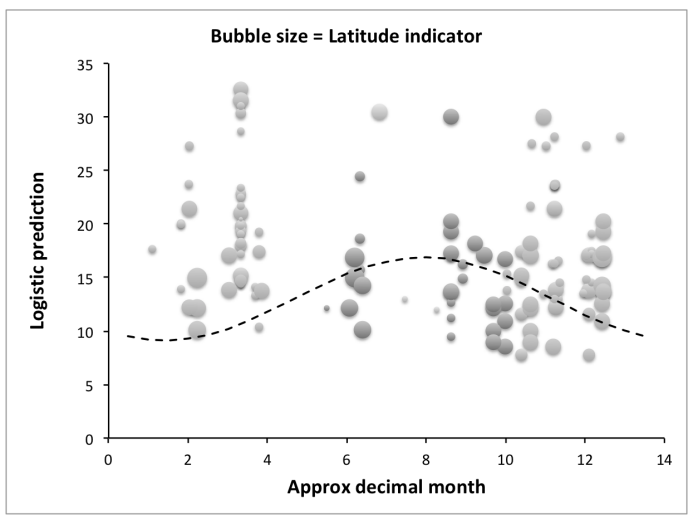

(a) Logistic prediction over locations across years. Dotted line is sinusoidal fit from Fig 7 with same phase and threshold but reduced gain (stiffness) showing reasonable fit ignoring winter outliers. Higher latitude $=$ larger bubble.

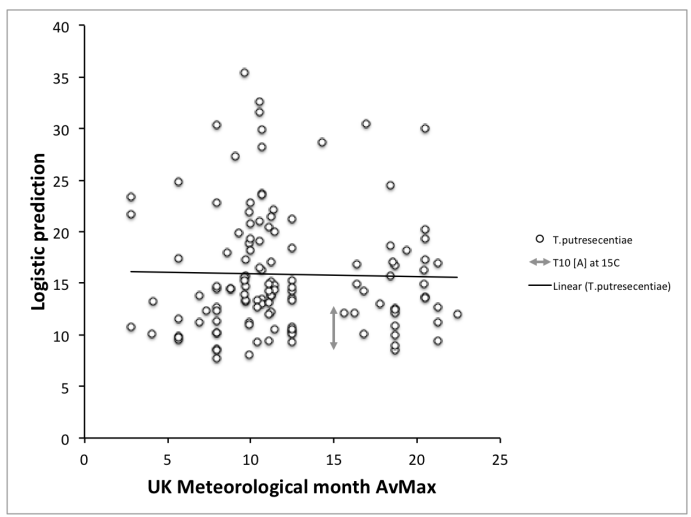

(b) Vertical spread indicates local not global ambient temperature confounds the general predictive relationship on top of considerable individual mite-to-mite variation. Grey arrow is range of predictions for independent laboratory culture figures abstracted from Owen (1978).

Figure 8. TRL5 UK test dataset mites (Tyrophagus putrescentiae males and females) logistic equation predicted temperature versus average monthly maximum for 3 closest (by road) UK Met Office Historic Station data or month over years of sampling. 


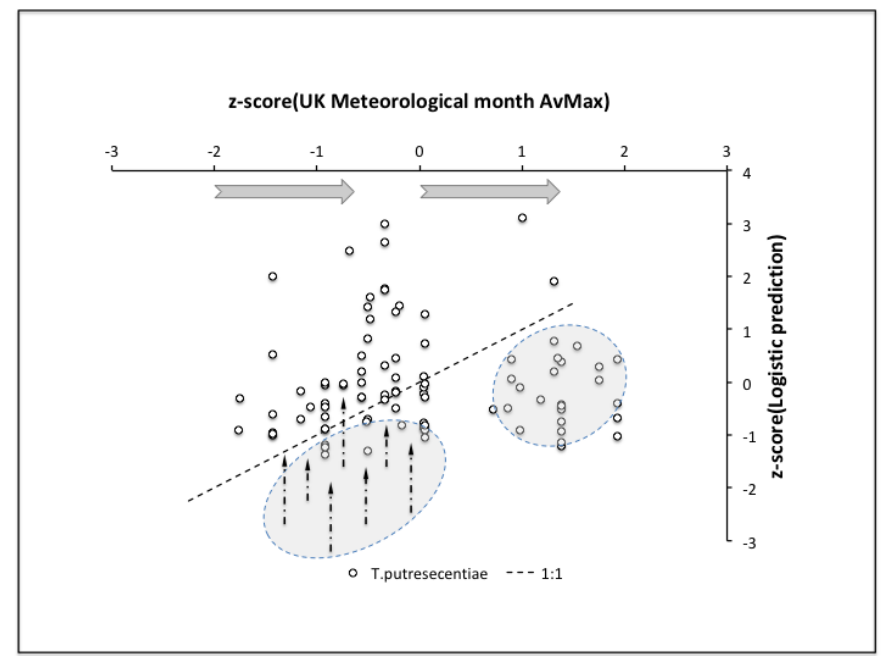

(a) Dual normalised data for easier comparison and summer mites set as ground 'truth' (equivalent to the horizontal axis being shifted right by about 1.5 units grey chevrons). This then infers winter collected mites should be in the approximate location of other empty grey elliptical zone to the left. These mites in the winter season are smaller than expected due to experiencing higher temperatures locally as indicated by little dashed arrows (see text for further explanation).

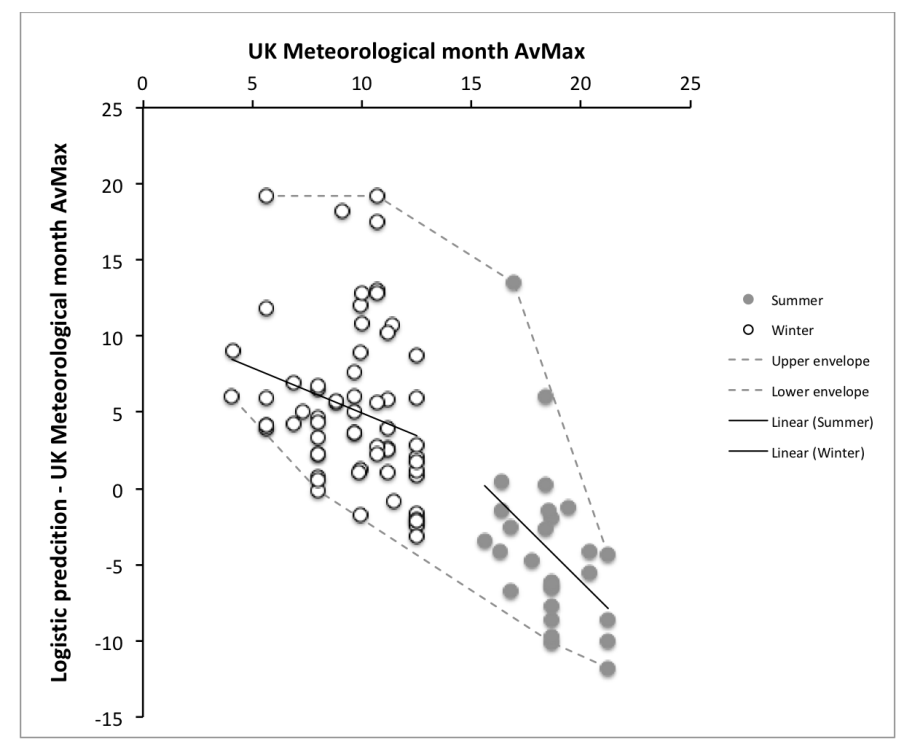

(b) Disparity between actual and predicted temperature by season showing that as temperatures fall local differential heating affects mite size more (note angle of slopes to regression lines suggesting a parabolic relationship overall) and expansion of the convex hull to the left ( = colder).

Figure 9. TRL5 UK test dataset mites (Tyrophagus putrescentiae males and females) logistic equation predicted temperature versus average monthly maximum for 3 closest (by road) UK Met Office Historic Station data. 


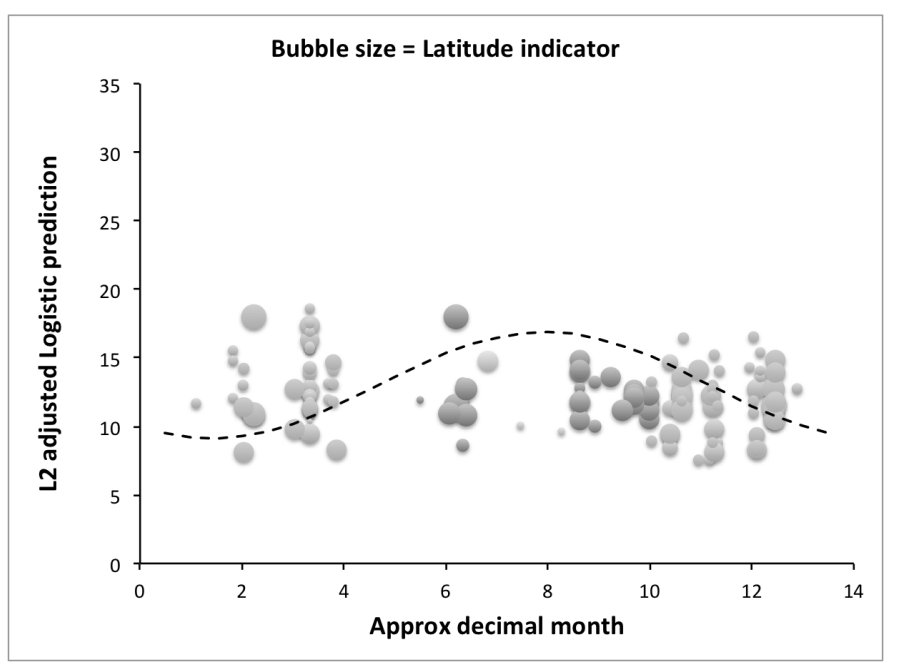

Figure 10. TRL5 UK test dataset mites (Tyrophagus putrescentiae males and females) logistic equation predicted temperature after linear adjustment with seta L2 length versus month over years. Note suppression of high outliers in winter, but essentially loss of overall signal seasonaliy. 